\title{
Adaptation of CAMP signaling system in SH-SY5Y neuroblastoma cells following expression of a constitutively active stimulatory G protein alpha, Q227L Gs $\alpha$
}

\author{
Ik-Soon Jang ${ }^{1}$ and Yong-Sung Juhnn ${ }^{1,2}$ \\ ${ }^{1}$ Department of Biochemistry and Cancer Research Institute, \\ Seoul National University College of Medicine, Seoul 110-799, \\ Korea \\ ${ }^{2}$ Corresponding author: Tel, +82-2-740-8247; Fax, +82-2-744-4534; \\ E-mail, juhnn@snu.ac.kr
}

Accepted 23 March, 2000

Abbreviations: $\mathrm{AC}$, adenylate cyclase; $\mathrm{ACl}, \mathrm{AC}$ isoform I; dbcAMP, dibutyryl CAMP; GAPDH, glyceraldehyde-3-phosphate dehydrogenase; Gs $\alpha$, alpha subunit of stimulatory G protein; PDE, cyclic nucleotide phosphodiesterase; PKA, CAMP-dependent protein kinase; RT-PCR, reverse transcription-polymerase chain reaction

\begin{abstract}
Heterotrimeric GTP-binding proteins (G protein) are known to participate in the transduction of signals from ligand activated receptors to effector molecules to elicit cellular responses. Sustained activation of CAMP-G protein signaling system by agonist results in desensitization of the pathway at receptor levels, however it is not clear whether such receptor responses induce other changes in post-receptor signaling path that are associated with maintenance of AMP levels, i.e. cAMP-forming adenylate cyclase (AC), cAMP-degrading cyclic nucleotide phosphodiesterase (PDE) and CAMP-dependent protein kinase (PKA). Experiments were performed to determine the expression of AC, PDE, and PKA isoforms in SHSY5Y neuroblastoma cells, in which cAMP system was activated by expressing a constitutively activated mutant of stimulatory G protein (Q227L Gs $\alpha$ ). Expression of ACI mRNA was increased, but levels of ACVIII and ACIX mRNA were decreased. All of the 4 expressed isoforms of PDE (PDE1C, PDE2, PDE 4A, and PDE4B) were increased in mRNA expression; the levels of PKA RI $\alpha$, RI $\beta$, and RII $\beta$ were increased moderately, however, those of RIl $\alpha$ and $\mathbf{C} \alpha$ were increased remarkably. The activities of AC, PDE and PKA were also increased in the SH-SY5Y cells expressing Q227L Gs $\alpha$. The similar changes in expression and activity of AC, PDE and PKA were observed in the SH-SY5Y cells treated with dbcAMP for 6 days. Consequently, it is concluded that the
\end{abstract}

cAMP system adapts at the post-receptor level to a sustained activation of the system by differential expression of the isoforms of AC, PDE, and PKA in SHSY5Y neuroblastoma. We also showed that an increase in cellular cAMP concentration might mediate the observed changes in the cAMP system.

Keywords: adenylate cyclase, cAMP-dependent protein kinase, 3',5'-cyclic-nucleotide phosphodiesterase, Protein isoforms, reverse transcriptase polymerase chain reaction

\section{Introduction}

Signal transducing heterotrimeric GTP-binding proteins ( $G$ protein) relay signals from activated receptors to intracellular effectors to elicit cellular responses (Gilman, 1987). Binding of signaling molecules such as hormones and neurotransmitters to the receptors makes the receptor undergo conformational change. This conformational change leads to activation of $G$ proteins by promoting the exchange of GDP for GTP on the G $\alpha$ subunit and finally the dissociation of G $\alpha$-GTP from its $G \beta \gamma$ dimer (Bourne et al., 1990). Both the dissociated Go-GTP and G $\beta \gamma$ subunits are able to mediate physiological responses by interacting with numerous effectors, including adenylate cyclase, phospholipases, phosphodiesterases, protein kinases, and ion channels (Neer, 1995). The G protein signal is terminated as the GTP on the G $\alpha$ subunit is hydrolyzed by intrinsic GTPase which is activated by the regulator of G protein-signaling (RGS) family (Zheng et al., 1999).

The cAMP system is one of the most intensively studied $\mathrm{G}$ protein signaling systems. Many extracellular signals including epinephrine and acetylcholine bind to and activate their seven transmembrane receptors, which couple to stimulatory $G$ proteins ( $G s \alpha$ ) or inhibitory $G$ proteins to regulate adenylate cyclase (AC, EC 4.6.1.1.) activity. Activated $A C$ catalyses the formation of $C A M P$, the key regulator of cAMP system, from ATP. However, the cellular concentration of CAMP is also regulated by cyclic nucleotide phosphodiesterases (PDE, EC 3.1.4.17.), which degrade cAMP into adenosine 5'-phosphate. An increase in CAMP concentration results in an activation of cAMP dependent protein kinase (PKA, EC 2.7.10.-) as well as ion channels and PKA, in turn, phosphorylates target proteins in order to regulate various metabolism 
and gene expression, resulting in numerous cellular responses, including proliferation, differentiation, and formation of memory.

When the cAMP signaling system is activated continuously by agonists, the desensitization of the pathway is induced at the receptor level by various mechanisms such as receptor phosphorylation, uncoupling of receptor and $\mathrm{G}$ proteins, down-regulation of the receptors (Ferguson and Caron, 1998). Sustained activation of cAMP system is also achieved, without activation of receptors, by expression of constitutively activated mutants of stimulatory G protein $(\mathrm{Gs} \alpha)$, and by treatment with forskolin or various cAMP analogues. Such sustained activation of CAMP system can influence the cAMP-forming AC, cAMP-degrading PDE and cAMP-binding PKA activity. Therefore, it is quite possible that the adaptation of cAMP system to the sustained activation can induce the downstream effectors such as AC, PDE, and PKA. However, the adaptation of $\mathrm{AC}, \mathrm{PDE}$, and PKA expression to a sustained activation of CAMP signaling system has not been clearly recognized.

At least nine genes for mammalian $A C$ have been cloned, and each isoform seems to have different kinetic and regulatory properties, including the response to calcium ions, beta gamma subunits of the $G$ protein, and protein kinase C (Sunahara et al., 1996). In mammals, PDE are encoded by at least 19 different genes, and each PDE gene contains several distinct transcriptional units that give rise to proteins with subtle structural differences, bringing the number of the PDE proteins close to 50 (Conti and Jin, 2000). Each PDE isoform seems to have different kinetic properties such as substrate specificity and it responds differentially to diverse regulators such as calcium/calmodulin and cyclic GMP. In addition, the expression of PDE isoforms varies in different tissues (Beavo, 1995). PKA is composed of distinct catalytic $(C)$ and regulatory $(R)$ subunits, and the holoenzyme contains two $C$ subunits bound to homo- or heterodimers of $R$ subunits. At present, 4 different $R$ subunits and three different $C$ subunits have been identified as separate gene products (Scott, 1991). The isoform multiplicity of AC, PDE and PKA, is suggested to play an important role in the regulation of the cAMP signaling system by intracellular targeting, crosstalk between a wide variety of other signaling systems, and flux-controlled sensitivity (Houslay and Milligan, 1997).

The present experiment was performed to examine how cAMP signaling system adapts at post-receptor levels to a sustained activation of cAMP system. The expression levels of isoforms of AC, PDE, and PKA were analyzed in SH-SY5Y neuroblastoma cells of which cAMP system was activated by expressing a constitutively activated mutant of Gs $\alpha$ (Q227L Gs $\alpha$ ) (Graziano and Gilman, 1989). We found that the expression of AC, PDE, and PKA isoforms were changed differentially to a sustained acti- vation of cAMP system resulting in adaptation at the post-receptor level.

\section{Materials and Method}

\section{Materials}

Materials were obtained as follows: isoproterenol, bovine serum albumin (BSA), Ethylene glycol-bis(beta-aminoethyl ether)-N,N,N',N'-tetraacetic acid (EGTA), N-(2-hydroxyethyl)piperazine-N'-(2-ethanesulfonic acid) (HEPES), and 3-isobutyl-1-methylxanthine (IBMX) were purchased from Sigma Chemicals; agarose, reverse transcriptase (Superscript II RNase $\mathrm{H}(-)$ Reverse transcriptase) were from GIBCO BRL; Taq polymerase was from TaKaRa; HA antibody against hemagglutinin peptide, GTP, FuGENE 6 Transfection Reagent, phosphocreatine, and creatine phosphokinase were from Roche Diagnostics. All other reagents were of analytical grade.

\section{Cell culture}

SH-SY5Y human neuroblastoma cells (CRL-2266) were purchased from American Type Culture Collection, and cells were cultured in Dulbecco's modified Eagle's medium containing $10 \%$ fetal calf serum, $100 \mathrm{IU} / \mathrm{ml}$ penicillin, and $50 \mu \mathrm{g} / \mathrm{ml}$ streptomycin in a humidified atmosphere containing $5 \% \mathrm{CO}_{2}$ in air at $37^{\circ} \mathrm{C}$.

\section{Establishment of SH-SY5Y cells expressing Q227L Gs $\alpha$}

A constitutively activated mutant of Gs $\alpha$, Q227L Gs $\alpha$ containing hemagglutinin (HA) epitope was kindly provided from Dr. H. Bourne (University of California San Francisco; Levis and Bourne, 1992). The gene was subcloned in pcDNA3 vector (Invitrogen), and the plasmid was transfected into $\mathrm{SH}-\mathrm{SY} 5 \mathrm{Y}$ neuroblastoma cells using FuGENE 6 Transfection Reagent. Transfected cells were grown in the medium containing $500 \mu \mathrm{g} / \mathrm{ml} \mathrm{G418.} \mathrm{The}$ clones expressing the mutant Gs $\alpha$ were selected by analyzing mRNA expression with reverse transcription-polymerase chain reaction (RT-PCR) using primers specific for HA tag region of Q227L Gs $\alpha$. The primers used for amplifying Gs $\alpha$ were as follows; for transfected Q227L Gs $\alpha$ 5'-GACGTGCCGGATTACGCGTCC and 5'-ATGAAGTACTTGGCCCGG, for total Gs $\alpha$ 5'-GCTGGAGAGTCTGGCAAAAGCACC and 5'-ATGAAGTACTTGGCCCGG. The expression of the mutant protein was also analyzed by Western blot using anti-HA antibody for detection of HA tag region of Q227L Gs $\alpha$ or RM antibody for detection of both the endogenous and exogenous Gs $\alpha$ protein as described (Yang et al., 1999).

\section{RNA preparation and semi-quantitative RT-PCR}

The total RNA was isolated from the harvested cells using acid guanidinium thiocyanate-phenol-chloroform 
extraction (Chomczynski and Sacchi, 1987). To analyze the relative amount of mRNA, a series of four mixtures was made by mixing the RNA from the control and the test sample in varying ratios, so that each reaction tube has the same total amount of RNA in a constant volume (Nicoletti and Sassy-Prigent, 1996). RT-PCR was performed to analyze the mRNA expression of $A C$ and PDE isoforms following the procedures described previously (Cho et al., 2000a; Cho et al., 2000b). Analysis of PKA isoform expression was performed in the similar way to that of $A C$ and $P D E$, and the primers used for amplifying PKA isoforms were as follows; for Rl $\alpha$ 5'AGGCAAAACAGATTCAG and 5'-CTCCTTCCCCAACACTG, for RI $\beta$ 5'-GAAGGTGATTCCCA AGGACTACAA and 5'-TCCCGGTCGATCCCCCAGAG, for RIl $\alpha$ 5'-CGACGCCAAAGGGGACAG and 5'-GGGGCACAGACTCAATAAATG, for RIIß 5'-CTGAACGCCTGAAAGTAGT$A G$ and 5'-GCAGTGGGTTCAACAATATCC, for $\mathrm{C} \alpha 5^{\prime}-$ AGTCCCGCTCAGAACACAG and 5'-GATAATCTCAGGGGCCAGG, for C $\beta$ 5'-TAATGCCCGGACTTGAAC and 5'-TCTGCCTTTAACTCTTT, for C $\gamma$ 5'-TACGCCGACCAGCCCATCCAG and 5'-AACCACAGGGGCACAAGCACA.

The amplified DNA products were separated on an agarose gel containing ethidium bromide, and the DNA band density was measured using a gel documentation system and the NIH Image $\mathrm{J}$ software. The amount of mRNA was calculated from a linear regression curve made of the measured density, and it was expressed as fold change relative to the vector-transfected control.

\section{Western blot analysis}

The expression of Gs $\alpha$ was analyzed by Western blot following the method described previously (Yang et al., 1998). The antibody against hemagglutinin peptide HA was used to detect HA-tagged mutant Gs $\alpha$ protein, while RM antibody raised against carboxy terminal peptide of Gs $\alpha$ was used to detect the total Gs $\alpha$ proteins, endogenous and transfected proteins.

\section{Measurement of cAMP accumulation}

From a 24-well plate containing the SH-SY5Y neuroblastoma cells, the culture medium was removed and then cells were incubated in $500 \mu \mathrm{l}$ of serum-free Dulbecco's modified Eagles medium containing $100 \mathrm{mM}$ IBMX with or without isoproterenol for $30 \mathrm{~min}$ at $37^{\circ} \mathrm{C}$. The treatment was terminated by aspiration of medium and by immediate addition of $2.5 \mathrm{M}$ perchloric acid, and the acid extracts were frozen at $-20^{\circ} \mathrm{C}$ until assay (Brown et al., 1971). After the acid extract was neutralized with 4.2 $\mathrm{M} \mathrm{KOH,} \mathrm{cAMP} \mathrm{levels} \mathrm{were} \mathrm{determined} \mathrm{by} \mathrm{competi-}$ tive binding with $\left[{ }^{3} \mathrm{H}\right]$ cAMP to the cAMP binding protein, which was prepared by expressing the regulatory subunit Rl $\alpha$ of CAMP dependent protein kinase in $E$. coli. Production of cAMP was normalized to the amount of acid-insoluble protein.

\section{Determination of enzyme activities}

AC

SH-SY5Y neuroblastoma cells were harvested and homogenized in ice-cold buffer containing $0.25 \mathrm{M}$ sucrose, 10 $\mathrm{mM}$ Tris- $\mathrm{HCl}$ (pH 7.5), $10 \mathrm{mM} \mathrm{MgCl}_{2}$, and 2 mM EGTA. Homogenates were centrifuged at $20,000 \mathrm{~g}$ at $4^{\circ} \mathrm{C}$ for $20 \mathrm{~min}$. Pellets were rehomogenized and washed twice with the above buffers, and resuspended in $10 \mathrm{mM}$ Tris$\mathrm{HCl}$ and $10 \mathrm{mM} \mathrm{MgCl} 2, \mathrm{pH}$ 7.4. The AC assay was carried out with fresh membranes stored in liquid nitrogen following the procedures described by Ammer and Schulz (Ammer and Schulz, 1997) with some modification. AC activity was determined in a reaction mixture $(100 \mu \mathrm{l})$ containing $40 \mathrm{mM}$ Tris- $\mathrm{HCl}, \mathrm{pH} 7.4,0.2$ mM EGTA, $100 \mathrm{mM} \mathrm{NaCl}, 10 \mathrm{mM} \mathrm{MgCl}$, $0.5 \mathrm{mM}$ ATP, $5 \mu \mathrm{g} / \mathrm{ml}$ phosphocreatine, $5 \mathrm{IU} / \mathrm{ml}$ creatine phosphokinase, $10 \mu \mathrm{M}$ GTP, and $0.5 \mathrm{mM}$ IBMX. Reactions were started by the addition of $15 \mu \mathrm{g}$ of membrane protein, allowed to continue for $10 \mathrm{~min}$ at $30^{\circ} \mathrm{C}$, and were stopped with $2.5 \mathrm{M}$ perchloric acid. The amount of cAMP generated was determined by CAMP binding assay (Brown et al., 1971).

\section{PDE}

PDE activity was determined by measuring the remaining cAMP in the assay mixture after the enzyme reaction. The reaction mixture was composed of $3 \mathrm{mM}$ $\mathrm{MgCl}_{2}, 60$ pmol cold cAMP, $1 \mathrm{mM}$ 5'-AMP, $50 \mathrm{mM}$ Tris$\mathrm{HCl}, \mathrm{pH} 7.4$ and cytosolic protein $(7-15 \mu \mathrm{g})$ in a final volume of $200 \mu \mathrm{l}$. The reaction was allowed for $15 \mathrm{~min}$ at $37^{\circ} \mathrm{C}$, and was stopped with $2.0 \mathrm{M}$ perchloric acid. The amount of remaining CAMP was determined by cAMP binding assay.

\section{PKA}

PKA activity was assayed as described previously (Yang et al., 1999). In brief, $10 \mu$ l of substrate cocktail containing $500 \mu \mathrm{M}$ Kemptide and $10 \mu \mathrm{M}$ cAMP, $10 \mu \mathrm{l}$ of inhibitor cocktail containing $20 \mu \mathrm{M}$ PKC inhibitor peptide and $20 \mu \mathrm{M}$ Compound R24571, and $10 \mu \mathrm{l}$ of tissue homogenate were added in order. Then the reaction was started by adding $10 \mu \mathrm{l}$ of the mixture containing $0.5 \mathrm{mM}$ ATP, $75 \mathrm{mM} \mathrm{MgCl} 2$ and $10 \mu \mathrm{Ci}$ of $\left[\gamma^{-32} \mathrm{P}\right] \mathrm{ATP}$ $(3000 \mathrm{Ci} / \mathrm{mmol})$, and the mixture were incubated for 10 minutes at $30^{\circ} \mathrm{C}$. After incubation, $25 \mu \mathrm{l}$ of the mixture was blotted on the P81 paper square, and the paper was washed once with $0.75 \%$ phosphoric acid. The assay square was transferred to a scintillation vial containing 5 $\mathrm{ml}$ scintillation cocktail, and its radioactivity was counted in a scintillation counter (Model Tri-Carb 1600 CA, Packard Instrument Company).

\section{Statistical analysis}

Results were analyzed using the two-tailed paired t-test, 
and a probability level of 0.05 or smaller was used for statistical significance. Results were expressed as the mean \pm standard deviation (SD). All experiments were repeated at least three times in duplicate or triplicate.
A

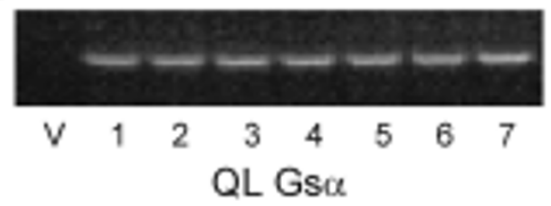

C

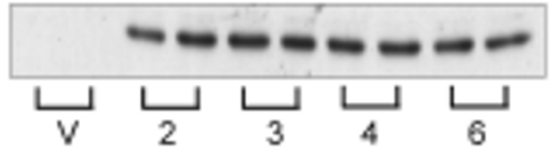

B

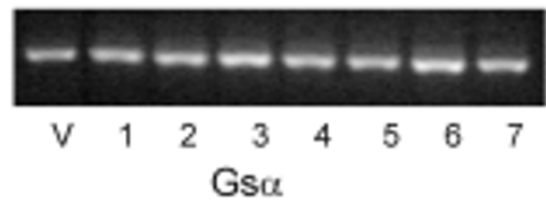

D

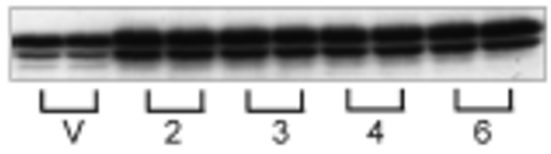

$\mathrm{Gs} \alpha$

Figure 1. Expression of Q227L Gs $\alpha$ in SH-SY5Y neuroblastoma cells. Expression of Q227L Gs $\alpha$ mRNA (A), total Gs $\alpha$ mRNA (B), Q227L Gs $\alpha$ protein (C) and total Gs $\alpha$ protein (D). Total RNA was prepared and reverse transcribed using oligo-dT primer, and resulting cDNA was amplified by PCR using primers specific for Q227L Gs $\alpha$ mRNA (A) or primers specific for total Gs $\alpha$ mRNA (B). The expression of the Gs $\alpha$ protein was analyzed by Western blots using an antibody detecting HA tag in the Q227L Gs $\alpha$ protein (C), or RM antibody detecting all forms of Gs $\alpha$ protein (D).

A

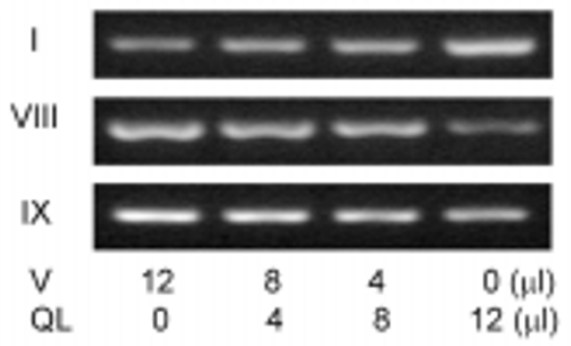

B
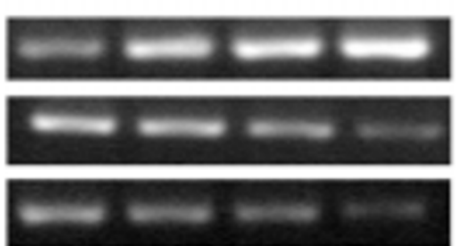

$\begin{array}{lllll}V & 12 & 8 & 4 & 0(\mu \mathrm{l})\end{array}$

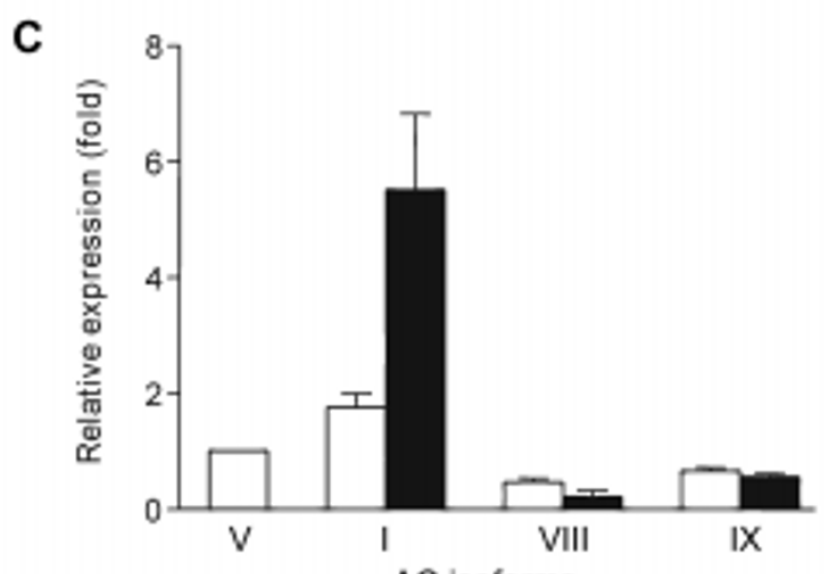

Figure 2. Expression of the AC isoform mRNA in SH-SY5Y neuroblastoma cells following over-expression of Q227L Gso or treatment with dbc AMP. Representative electropherograms of RT-PCR product of AC isoforms in cells expressing Q227L Gs $\alpha$ (A) and in dbcAMP-treated cells (B). (C) Summary of mRNA expression of AC isoforms. Empty bars represent the expression in cells expressing Q227L Gso, and filled bars represent the expression in the dbcAMP treated cells. The total RNA was isolated from the cells expressing Q227L Gs $\alpha$ or cells treated with dbcAMP for 6 days using acid guanidinium thiocyanatephenol-chloroform extraction. Series of four mixtures were prepared by mixing the RNA with the control and the test sample in varying ratios, so that each reaction tube has the same total amount of RNA in a constant volume to measure the relative amount of mRNA. Semi-quantitative RT-PCR was performed using AC isoform-specific primer sets. The amount of mRNA was calculated from a linear regression curve made from measured density. Values presented are the mRNA amounts expressed as a fold of the mRNA of the vector transfected controls, and are the mean \pm standard deviation of at least 3 independent experiments. All the data presented displayed statistically significant difference from the control group $(p<0.05$, paired t-test, two-tailed) 


\section{Results}

\section{Expression of constitutively activated Q227L Gs $\alpha$}

SH-SY5Y neuroblastoma cells transfected with Q227L Gs $\alpha$ were grown in the presence of $G 418$, and then clones expressing mRNA of Q227L Gs $\alpha$ were selected by RT-PCR analysis of mRNA containing HA sequence. Most of the clones expressed Q227L Gs $\alpha$ mRNA containing HA tag sequence, in contrast to the vector-transfected control that did not show the expression (Figure $1 \mathrm{~A})$. The segment of wild type Gs $\alpha$ was amplified in both the vector control and mutant expressing cells (Figure 1B). The expression of Q227L Gs $\alpha$ protein of the selected clones was further confirmed by Western blot probed using anti-HA antibody (Figure 1C), which indicated that the mutant tagged proteins were expressed. Western blot analysis using RM antibody, which detected both the wild and mutant types of the protein, showed about $50 \%$ increase in the total immunoreactivity in the selected clones (Figure 1D). The increase in the immunoreactivity was observed in both the long forms and short forms of Gs $\alpha$ together. Two clones were selected and used for further analysis in this experiment.

\section{Expression of AC, PDE, and PKA isoform mRNAs}

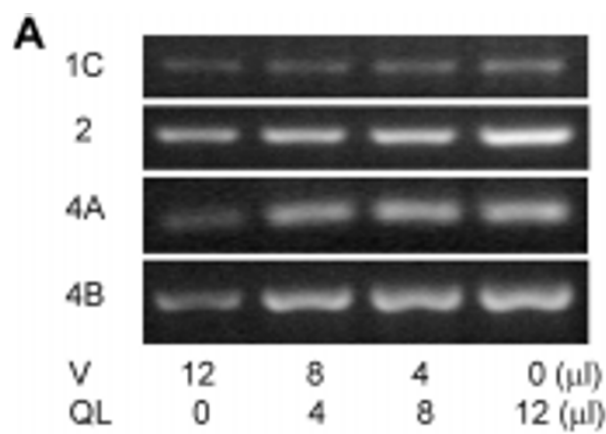

In SH-SY5Y cells, ACl, ACVIII, and ACIX mRNAs, among $9 \mathrm{AC}$ isoforms, were expressed enough for quantitative RT-PCR analysis. In the Q227L Gs $\alpha$-expressing SHSY5Y neuroblastoma cells, the expression of $\mathrm{ACl}$ increased to $178 \%$, but that of ACVIII and ACIX was decreased respectively to $44 \%$ and $66 \%$ of the vector transfected control cells. When SH-SY5Y neuroblastoma cells were treated with dbcAMP for 6 days, the expression of $A C$ isoforms changed similarly, that is, $\mathrm{ACl}$ increased more prominently to $551 \%$ and ACVIII and $\mathrm{ACIX}$ decreased to $21 \%$ and $56 \%$ of the untreated control, respectively (Figure 2).

In SH-SY5Y neuroblastoma cells, PDE 1C, 2, 4A, and $4 B$ were expressed enough amount for analysis by the RT-PCR method. All of the 4 PDE isoform increased in mRNAs expression significantly; PDE1C to $328 \%$, PDE2 to $207 \%$, PDE $4 \mathrm{~A}$ to $390 \%$, and PDE4B to $272 \%$. The similar increasing pattern of the PDE isoform expression was observed in the SH-SY5Y neuroblastoma cells treated with dbcAMP for 6 days. The expression of PDE1C increases to $388 \%$, PDE2 to $539 \%$, PDE4A to $683 \%$, and PDE4B to $493 \%$ respectively in the cells treated with dbcAMP for 6 days (Figure 3 ).

All of the 4 isoforms of PKA regulatory subunit were expressed in SH-SY5Y neuroblastoma cells, but $\mathrm{C} \alpha$ subunit was the only isoform expressed in the cells.

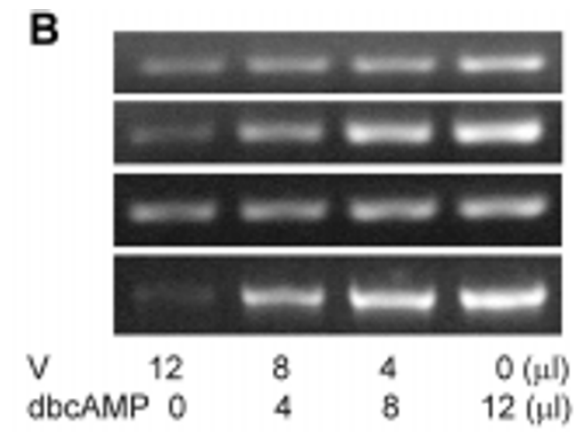

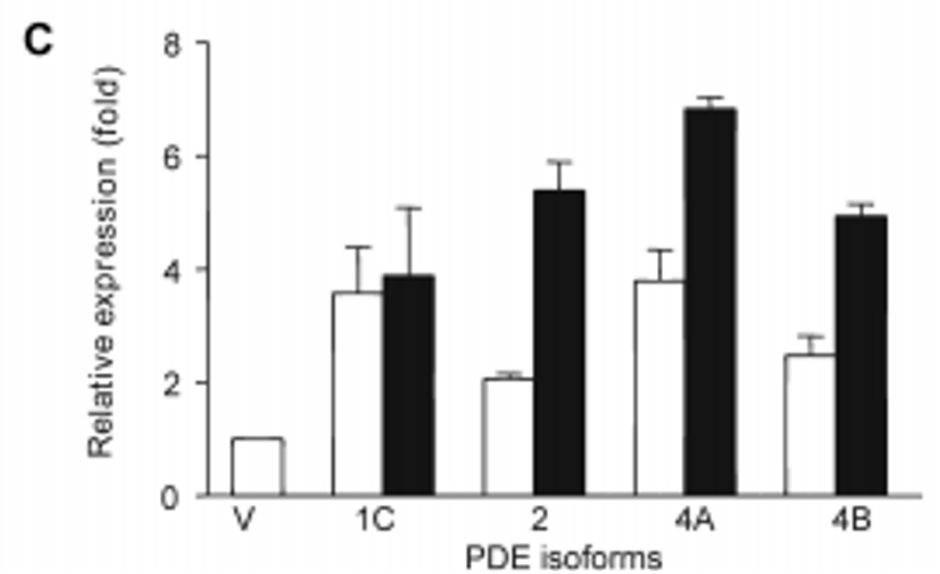

Figure 3. Expression of the PDE isoform mRNA in SH-SY5Y neuroblastoma cells following over-expression of Q227L Gso. Representative electropherograms of RT-PCR product of PDE isoforms in cells expressing Q227L Gs $\alpha(A)$ and in dbcAMP-treated cells (B). (C) Summary of mRNA expression of PDE isoforms. Empty bars represent the expression in cells expressing Q227L Gs $\alpha$, and filled bars the expression in the dbcAMP treated cells. Refer to the legend of Figure 2. 
A

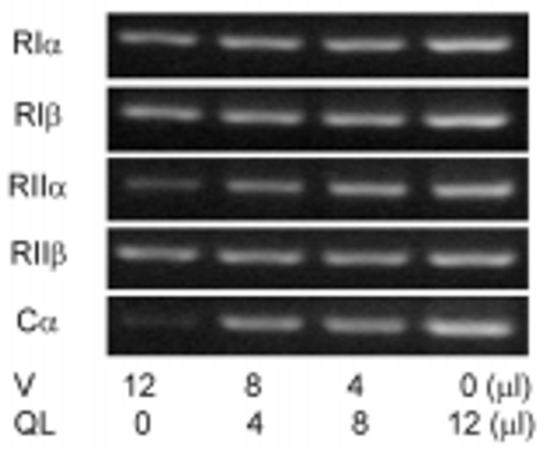

B

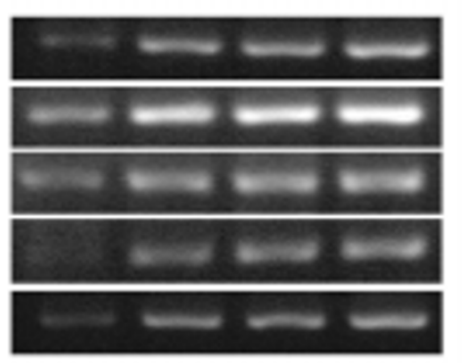

$\begin{array}{lllll}V & 12 & 8 & 4 & 0(\mu \mathrm{l})\end{array}$

QL $\quad \begin{array}{llll}0 & 4 & 8 & 12(\mu))\end{array}$

C

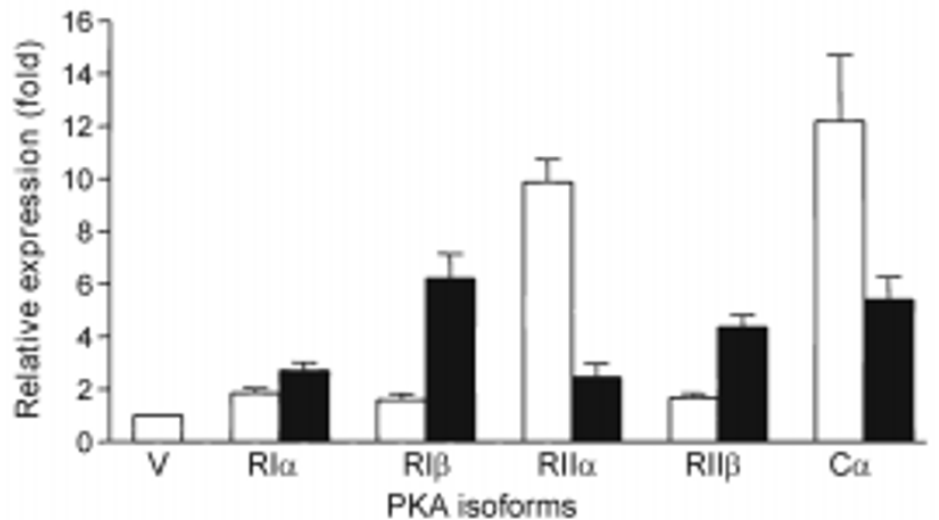

Figure 4. Expression of the PKA isoform mRNA in SH-SY5Y neuroblastoma cells following over-expression of Q227L Gso. Representative electropherograms of RT-PCR product of PKA isoforms in cells expressing Q227L Gs $\alpha$ (A) and in dbcAMP treated cells (B). (C) Summary of mRNA expression of PKA isoforms. Empty bars represent the expression in cells expressing Q227L Gs $\alpha$, and filled bars the expression in the dbcAMP treated cells. Refer to the legend of Figure 2.

Although the expression levels of the PKA regulatory subunits such as $R|\alpha, R| \beta$ and $R \| I \beta$ were increased in the range of $160 \%$ to $187 \%$, that of Rllo was increased most prominently to 9.9-fold compared the control. The expression of $\mathrm{C} \alpha$ was also remarkably increased to 12fold to the vector transfected control. Treatment of the neuroblastoma cells with dbcAMP for 6 days also resulted in the increase of all PKA subunit isoforms expression. The expression of Rl $\alpha$ was increased to $270 \%$, $\mathrm{RI} \beta$ to $621 \%$, Rll $\alpha$ to $244 \%$, RII $\beta$ to $436 \%$, and $\mathrm{C} \alpha$ to $540 \%$ of the untreated control (Figure 4).

\section{Activity of AC, PDE and PKA}

To determine the effects of the changes in the mRNA expression of AC, PDE, and PKA, the activity of each enzyme was assayed. Both the basal and forskolinstimulated AC activity was increased in Q227L Gs $\alpha$ transfected SH-SY5Y neuroblastoma cells. The basal AC activity of Q227L Gs $\alpha$ expressing cells increased by 4.1fold $(12.7 \pm 0.6 \mathrm{pmol} / \mathrm{min} \mathrm{mg}$-protein) from the control (3.1 \pm 1.3$)$, and forskolin activated AC activity was also increased from $95.4 \pm 8.2$ to $134.0 \pm 15.2$. The PDE activity was also increased about 2.4-fold in Q227L Gs $\alpha$

Table 1. Activity of AC and PKA in SH-SY5Y neuroblastoma cells expressing Q227L Gs $\alpha$. The AC activity was determined by the fresh membranes prepared from the Q227L Gs $\alpha$ expressing cells and vector transfected cells, and asterisk $\left({ }^{*}\right)$ indicates AC activity stimulated by forskolin. The PDE activity was determined by measuring remaining cAMP in the assay mixture, and the PKA activity was determined in cell homogenates with Kemptide and $\left[\gamma^{32}\right.$ P]ATP as substrates in the presence of $100 \mu \mathrm{M}$ cAMP. The enzyme activity was expressed as $\mathrm{pmol} / \mathrm{min} \mathrm{mg}-$ protein

\begin{tabular}{lccc}
\hline & $\begin{array}{c}\text { AC activity } \\
\text { (pmol/min mg-protein) }\end{array}$ & $\begin{array}{c}\text { PDE activity } \\
\text { (pmol/min mg-protein) }\end{array}$ & $\begin{array}{c}\text { PKA activity } \\
\text { (pmol/min mg-protein) }\end{array}$ \\
\hline Vector transfected & $3.1 \pm 1.3$ & $89.3 \pm 20.2$ & $14.4 \pm 0.9$ \\
Q227L Gs $\alpha$ transfected & $95.4 \pm 8.2^{*}$ & $212.8 \pm 29.8$ & $50.5 \pm 2.6$ \\
DbcAMP treated & $12.7 \pm 0.6$ & $308.7 \pm 16.2$ & $53.7 \pm 2.8$ \\
\hline
\end{tabular}


expressing cells $(212.8 \pm 29.8 \mathrm{pmol} / \mathrm{min} \mathrm{mg}$-protein $)$ compared the control $(89.3 \pm 20.2)$. The activity of PKA was increased about 3.5-fold from the vector-transfected control (from $14.4 \pm 0.9 \mathrm{pmol} / \mathrm{min}$ mg-protein to $50.5 \pm$ 2.6) (Table 1).

The similar changes in the activities of AC and PKA were also observed in the neuroblastoma cells treated with dbcAMP for 6 days (Table 1). The basal (17.2 \pm 3.5 $\mathrm{pmol} / \mathrm{min} \mathrm{mg}$-protein) and forskolin stimulated (182 \pm 12.1) AC activity increased more in dbcAMP-treated cells than Q227L Gs $\alpha$ expressing cells (Table 1).

\section{cAMP response}

To study the consequence of the changes in the mRNA and protein expression of $A C$ and PDE, we analyzed the cellular cAMP response. The basal cAMP level was increased from $8.6 \pm 0.3 \mathrm{pmol} / \mathrm{mg}$ protein to $71.6 \pm 3.3$ $\mathrm{pmol} / \mathrm{mg}$ protein in Q227L Gs $\alpha$ transfected SH-SY5Y cells, and the cAMP response to isoproterenol stimulation was also increased from $54.0 \pm 4.9$ to $121 \pm 5.6$ $\mathrm{pmol} / \mathrm{mg}$ protein. These data showed that both the basal and isoproterenol-stimulated accumulation of cAMP increased in the Q227L Gs $\alpha$ expressing cells. Similar increases in CAMP response were observed in the $\mathrm{SH}$ SY5Y neuroblastoma cells treated with dbcAMP for 6 days. The cAMP level was $72.6 \pm 6.3 \mathrm{pmol} / \mathrm{mg}$ protein at basal and $161 \pm 7.1 \mathrm{pmol} / \mathrm{mg}$ protein at stimulated state (Table 2).

\section{Discussion}

This study was performed to examine how cAMP signaling system adapts to a sustained activation of cAMP system at post-receptor levels, and it demonstrated that cAMP system adapts to its sustained activation, by differential changes of isoform expression of $A C$, PDE,

Table 2. cAMP response in SH-SY5Y neuroblastoma cells expressing Q227L Gs $\alpha$. From the SH-SY5Y neuroblastoma cells in a 24-well plate, cells were incubated in $500 \mu \mathrm{l}$ serum-free Dulbecco's modified Eagle's medium containing $100 \mu \mathrm{M} \mathrm{IBMX}$ with or without isoproterenol for $30 \mathrm{~min}$ at $37^{\circ} \mathrm{C}$. The cAMP level in perchloric acid extract was determined by competitive binding with [ $\left.{ }^{3} \mathrm{H}\right] \mathrm{CAMP}$ to a cAMP binding proteins, and it was normalized to the amount of acid-insoluble protein

\begin{tabular}{lc}
\hline & cAMP \\
& $(\mathrm{pmol} / \mathrm{mg}$ protein $)$ \\
\hline Vector transfected & \\
unstimulated & $8.6 \pm 0.3$ \\
$\quad$ Isoproterenol stimulated & $54.0 \pm 4.9$ \\
Q227L Gs $\alpha$ transfected & \\
$\quad$ unstimulated & $71.6 \pm 3.3$ \\
$\quad$ Isoproterenol stimulated & $121 \pm 5.6$ \\
DbcAMP treated & \\
$\quad$ unstimulated & $72.6 \pm 6.3$ \\
Isoproterenol stimulated & $161 \pm 7.1$ \\
\hline
\end{tabular}

and PKA isoforms in SH-SY5Y neuroblastoma cells expressing Q227L Gs $\alpha$.

Among the $A C$ isoforms analyzed in this study, expression of $\mathrm{ACl}$ increased while expression of $\mathrm{ACVII}$ and $A C I X$ decreased. The change in mRNA expression resulted in the increase of forskolin-stimulated activity by 1.4fold in Q227L Gs $\alpha$ expressing SH-SY5Y neuroblastoma cells. This result indicates that increase in AC activity is a net result of increase in $\mathrm{ACl}$ and concomitant decrease in ACVIII and ACIX. Besides, each AC isoform is different in their kinetic and regulatory properties (Simonds, 1999). The activity of ACl is inhibited by inhibitory $\mathrm{G}$ protein alpha subunit and beta-gamma subunit complex of $G$ protein, but it is activated by calcium/ calmodulin complex and protein kinase $\mathrm{C}$. The activity of ACVIII is also activated by calcium/calmodulin complex while the activity of ACIX is inhibited by calcium ions. Thus, increase in the expression of $\mathrm{ACl}$ isoform in $\mathrm{SH}$ SY5Y neuroblastoma cells following expression of Q227L Gs $\alpha$ may also result in the increase of the calcium sensitivity and inhibition by G proteins of cAMP signaling system.

Similar to $A C$ isoforms, each PDE isoform has different kinetic and regulatory properties. PDE1 family is a calcium/calmodulin dependent enzyme, and has a substrate preference for cGMP. PDE2 is cGMP-stimulated enzyme, which increase PDE2 activity by allosteric mechanism. Thus this isoform is implicated to synchronize the cGMP and cAMP signaling system (Beavo, 1995). PDE4 family is currently the largest PDE family, and it is widely distributed in many tissues. Because PDE4 isoform is cAMP-specific, it is usually the major enzyme responsible for degradation of cAMP. Therefore, the increased expression of PDE1 observed in the present study might increase calcium/calmodulin dependency of the system, while increased PDE2 expression might increase in cGMP dependency. Increased activity of PDE4A and 4B might contribute to the rapid removal of CAMP formed by AC stimulated by expressed Q227L Gs $\alpha$. Similar adaptation of two PDE isoforms was reported in the rat Sertoli cell, where the prolonged stimulation of CAMP system by $\mathrm{FSH}$, forskolin, cholera toxin, and N6, O2'dibutyryl cAMP increased in PDE3 and PDE4 expression (Swinnen et al., 1991).

The large increase in RIl $\alpha$ and $\mathrm{C} \alpha$ isoform of PKA mRNA expression observed here by expressing Q227L Gs $\alpha$, also resulted in the increase of the enzyme activity. A high level expression of PKA RI is correlated with active cell growth and transformation, in contrast to high-level expression of RII, which is correlated with growth arrest and differentiation (Cho-Chung, 1990). Similar changes were obtained by treating cancer cells with 8-Cl-cAMP, a cAMP analogue, which showed a decrease of Rl $\alpha$, an increase of RII $\beta$, and inhibition of cell growth (Cho-Chung, 1990).

The mRNA levels do not always reflect the levels of 
protein in the examined cells and tissues. Changes in mRNA expression of AC, PDE and PKA isoforms resulted in the changes of respective enzyme activity. The net result of such differential changes in isoform mRNA expression would primarily be determined by the relative contribution of each isoform to the total enzyme activity in a cell or subcellular localization, and by the extent to which individual isoform expression altered. Thus, an analysis of the relative contribution of each isoform to the total activity is essential to understand the functional significance of changes in AC isoform expression. Therefore, better correlation between mRNA and enzyme activity could be obtained by studies such as isoform specific enzyme activity assay following immunoprecipitation with isoform-specific antibody, and the analysis of isoform specific enzyme expression by quantitative Western blot.

We found that the basal and isoproterenol stimulated cAMP response were increased in SH-SY5Y neuroblastoma cells following the expression of Q227L Gso. The increase in cAMP accumulation may have resulted from an increased AC activity, a decreased PDE activity, or both. The increase in the expression and the activity of both AC and PDE were observed in the present study. Increase in the activity of both cAMP forming AC and cAMP-degrading PDE makes the cell to produce more cAMP and to degrade cAMP more rapidly, so-called high cAMP cycling condition. Such system has high basal $A C$ and PDE activity so that either activation of $A C$ or inhibition of PDE can activate PKA by elevation of cAMP level above the threshold concentration. Before adaptation, the SHSY-5Y cells have low AC and low PDE activity, and the PKA activation threshold is unlikely breached by PDE inhibition in such cells. Thus, in low cAMP cycling condition, AC activation dominates as the means for triggering responses, and it takes longer time to achieve maximum cAMP responses (Houslay and Milligan, 1997). Thus, SH-SY5Y cells seem to have adapted to the expression of Q227L Gs $\alpha$ from low cAMP cycling condition to high cycling condition. This adaptation demonstrates that the regulatory properties of the cAMP system can be regulated by differential expression of the isoforms of $A C$ and PDE. Such changes in cAMP system may result in the changes of cellular response induced by many biological signals that act through or cross talk with cAMP systems.

In order to examine the mechanism for the differential expression of the isoforms of AC, PDE and PKA induced by expression of Q227L Gs $\alpha$ in SH-SY5Y neuroblastoma cells, we analyzed the expression of the enzyme isoforms in the cells treated with dbcAMP, a membrane permeable cAMP analogue. Similar patterns of isoform expression, though not the same, were observed in the dbcAMP treated cells and Q227L Gs $\alpha$ expressing SHSY5Y neuroblastoma cells. This result suggests that the differential expression of the isoforms in Q227L Gs $\alpha$ ex- pressing cells is mediated by an increase in intracellular cAMP. Greater increase of AC mRNA and activity in the dbcAMP treated cells than Q227L Gs $\alpha$ expressing cells might reflect the shorter duration of cAMP system activation than Q227L Gs $\alpha$ expressing cells.

Mutant Q227L Gs $\alpha$ differs from the wild type protein in the substitution of Leu for Gln227, respectively. The rate constant for hydrolysis of GTP by Q227L Gs $\alpha$ is roughly 100 -fold slower than that of wild type Gs $\alpha$. This reduction in the rate of hydrolysis of GTP results in significant fractional occupancy of this protein by GTP in the presence of the nucleotide, and causes constitutive activation of the protein (Graziano and Gilman, 1989). Thus this Q227L Gs $\alpha$ is widely used to activate cAMP signaling system in various tissues (Schutzer et al., 2000). The cAMP system was found in this study to induce adaptive response to the continuous activation caused by expression of Q227L Gs $\alpha$ by differential expression of isoforms of AC, PDE, and PKA in SH-SY5Y neuroblastoma cells.

In conclusion, we have shown that cAMP system adapts to a sustained activation of the system, by differential expression of the isoforms of AC, PDE, and PKA at mRNA and by changing enzyme activity in SH-SY5Y neuroblastoma cells expressing Q227L Gso. We have also shown that an increase in cellular cAMP concentration is involved in this adaptation process. The adaptation of cAMP system is suggested to change the regulatory properties of the system so that it may change many signaling system acting through the cAMP system to modify various cellular responses.

\section{Acknowledgement}

This work was supported by grant No. (971-0708-059-2) from the Basic Research Program of the Korea Science \& Engineering Foundation and by Basic Medical Research Fund of Ministry of Education, Republic of Korea (1996).

\section{References}

Ammer, H. and Schulz, R. (1997) Enhanced stimulatory adenylyl cyclase signaling during opioid dependence is associated with a reduction in palmitoylated Gs alpha. Mol. Pharmacol. 52: 993-999

Beavo, J. A. (1995) Cyclic nucleotide phosphodiesterases: functional implications of multiple isoforms. Physiol. Rev. 75: 725-748

Bourne, H. R., Sanders, D. A. and McCormick, F. (1990) The GTPase superfamily: a conserved switch for diverse cell functions. Nature 348: 125-132

Brown, B. L., Albano, J. D., Ekins, R. P. and Sgherzi, A. M. (1971) A simple and sensitive saturation assay method for the 
measurement of adenosine $3^{\prime}, 5^{\prime}$-cyclic monophosphate. Biochem. J. 121: 561-562

Cho, C. H., Cho, D. H., Seo, M. R. and Juhnn, Y. S. (2000a) Differential changes in the expression of cyclic nucleotide phosphodiesterase isoforms in rat brains by chronic treatment with electroconvulsive shock. Exp. Mol. Med. 32: 110-114

Cho, D.-H., Bae, C.-D. and Juhnn, Y.-S. (2000b) Differential expression of adenylate cyclase Isoforms in B16-F10 melanoma cells differentiated with forskolin. Exp. Mol. Med. 32: 235-242.

Cho-Chung, Y. S. (1990) Role of cyclic AMP receptor proteins in growth, differentiation, and suppression of malignancy: new approaches to therapy. Cancer Res. 50: 7093-7100

Chomczynski, P. and Sacchi, N. (1987) Single-step method of RNA isolation by acid guanidinium thiocyanate-phenol-chloroform extraction. Anal. Biochem. 162: 156-159

Conti, M. and Jin, S. L. (2000) The molecular biology of cyclic nucleotide phosphodiesterases. Prog. Nucleic Acid. Res. Mol. Biol. 63: 1-38

Ferguson, S. S. and Caron, M. G. (1998) G protein-coupled receptor adaptation mechanisms. Semin. Cell Dev. Biol. 9: 119-127

Gilman, A. G. (1987) G-proteins: transducers of receptorgenerated signals. Annu. Rev. Biochem. 56: 615-649

Graziano, M. P. and Gilman, A. G. (1989) Synthesis in Escherichia coli of GTPase-deficient mutants of Gs alpha. J. Biol. Chem. 264: 15475-15482

Houslay, M. D. and Milligan, G. (1997) Tailoring cAMP-signalling responses through isoform multiplicity. Trends Biochem. Sci. 22: 217-224

Levis, M. J. and Bourne, H. R. (1992) Activation of the alpha subunit of $G$ s in intact cells alters its abundance, rate of degradation, and membrane avidity. J. Cell Biol. 119: 12971307
Neer, E. J. (1995) Heterotrimeric G proteins: organizers of transmembrane signals. Cell 80: 249-257

Nicoletti, A. and Sassy-Prigent, C. (1996) An alternative quantitative polymerase chain reaction method [published erratum appears in Anal Biochem 1996; 242(1):164]. Anal. Biochem. 236: 229-241

Schutzer, W. E., Watts, V. J., Chapman, J., Cumbay, M. G., Neve, K. A., Neve, R. L. and Mader, S. L. (2000) Viral-mediated gene delivery of constitutively activated $G$ alpha $S$ alters vasoreactivity. Clin. Exp. Pharmacol. Physiol. 27: 9-13

Scott, J. D. (1991) Cyclic nucleotide-dependent protein kinases. Pharmacol. Ther. 50: 123-145

Simonds, W. F. (1999) G protein regulation of adenylate cyclase. Trends Pharmacol. Sci. 20: 66-73

Sunahara, R. K., Dessauer, C. W. and Gilman, A. G. (1996) Complexity and diversity of mammalian adenylyl cyclases. Annu. Rev. Pharmacol. Toxicol. 36: 461-480

Swinnen, J. V., Tsikalas, K. E. and Conti, M. (1991) Properties and hormonal regulation of two structurally related CAMP phosphodiesterases from the rat Sertoli cell. J. Biol. Chem. 266: $18370-18377$

Yang, J. M., Cho, C. H., Bae, C. D. and Juhnn, Y. S. (1998) Retainment of membrane binding capacity of non-palmitoylated Gs alpha mutants expressed in COS-1 cells. Exp. Mol. Med. 30: 235-239

Yang, J. M., Cho, C. H., Kong, K. A., Jang, I. S., Kim, H. W. and Juhnn, Y. S. (1999) Increased expression of Galphaq protein in the heart of streptozotocin-induced diabetic rats. Exp. Mol. Med. 31: 179-184

Zheng, B., De Vries, L. and Gist Farquhar, M. (1999) Divergence of RGS proteins: evidence for the existence of six mammalian RGS subfamilies. Trends Biochem. Sci. 24: 411414 